\title{
Knowledge and Perceptions of People Living With HIV Regarding The HIV Services That Are Offered By The Community Health Workers in KwaZulu-Natal, South Africa
}

Gugulethu Eve Khumalo ( $\nabla$ jcatcalvary@gmail.com )

University of KwaZulu-Natal

Bontle Segobe

Department of Health, KwaZulu-Natal

Elizabeth Lutge

Department of Health, KwaZulu-Natal

Tivani P Mashamba-Thompson

University of Pretoria

\section{Research Article}

Keywords: people living with HIV, HIV knowledge and perceptions, community health worker, HIV services

Posted Date: March 1st, 2021

DOl: https://doi.org/10.21203/rs.3.rs-246112/v1

License: (c) (i) This work is licensed under a Creative Commons Attribution 4.0 International License.

Read Full License 


\section{Abstract}

Background: KwaZulu-Natal (KZN) is the province with the highest HIV prevalence in South Africa (SA). Community Health Workers (CHWs) are key to delivery of HIV services at community level. Evidence on knowledge and perceptions of people living with HIV (PLWH) regarding the HIV services that are offered by the CHWs is limited. Therefore, this study seeks to determine knowledge and perceptions of PLWH regarding the HIV services that are offered by the CHWs in KZN.

Methods: The study design was a quantitative survey using an administrator-administered questionnaire. A total of 303 PLWH from 3 selected KZN clinics were interviewed to determine their knowledge and perceptions of HIV services offered by the CHWs. Statistical Package for Social Sciences (SPSS) version 27 was used to describe the population and testing for associations between variables. The significant level was set at a p value $\leq 0.05$ and at $95 \%$ confidence internal.

Results: Among the 303 PLWH surveyed, 24 (8\%), knew about the HIV services offered by the CHWs and of the $89(29 \%)$ participants that were visited by CHWs, 73 (82\%) had a positive perception about these services. Participants who were visited by a CHW were more likely (OR=1.57, 95\% Cl: 0.57-4.35) to know about the HIV services. Knowledge of HIV services was significantly associated with the positive perception of the HIV services $(p<0.05)$. Knowledge and perception of the HIV services was not associated with age, gender, level of education or duration of visiting the clinic.

Conclusion: The majority of PLWH in KZN have poor knowledge of HIV services offered by the CHWs and most of them have never been visited by a CHW in their homes. Those that were visited by CHWs were more likely to have positive perceptions regarding their HIV services. The findings of the study should trigger the scaling up of HIV community targeted initiatives that are delivered by CHWs in order to curb the HIV epidemic in the province.

\section{Introduction}

KwaZulu-Natal (KZN) remains the province with the highest prevalence of Human Immunodeficiency Virus (HIV) in South Africa (SA) ${ }^{[1-3]}$. Community health workers (CHWs) have contributed to the strengthening of the HIV programme by linking people living with HIV (PLWH) to HIV healthcare, promoting ART adherence, and improving retention in care ${ }^{[4-7]}$. However, antiretroviral treatment (ART) uptake is poor in $\mathrm{KZN}$ and this is related to multiple psychosocial, socioeconomic and socio-medical barriers that act at many levels including the level of the individual ${ }^{[8-9]}$. In KZN, PLWH have mixed feelings about CHWs, where some are satisfied with the HIV services they provide in the communities [10] and some do not trust them due to their perceived lack of confidentiality ${ }^{[11]}$.

The roles of $\mathrm{CHWs}$ have been expanded as a strategy to strengthen HIV healthcare services and provide longitudinal patient support in KZN ${ }^{[5,8,12]}$. Furthermore community HIV interventions by CHWs have been seen as an imnortant nredictor of HIV treatment success ${ }^{[13]}$. The expansion is also part of the primary Loading [MathJax]/jax/output/CommonHTML/fonts/TeX/fontdata.js 
healthcare $(\mathrm{PHC})$ re-engineering programme in SA that culminated in the launch of Ward Based Primary Healthcare Outreach Teams (WBPHCOT) framework policy in 2018 for which CHWs are the key components ${ }^{[14]}$. In 2016, the KZN Department of Health (KZN-DOH) also implemented the universal test and treat (UTT) strategy for meeting the 90-90-90 targets of the United Nations Programme on HIV/AIDS (UNAIDS), and to ensure that PLWH continue to take ART and therefore achieve viral suppression [15]. However the UTT targets are off track and have not been met in the province ${ }^{[16-19]}$. In spite of the community interventions through the HIV services provided by CHWs in KZN, ART uptake, which is the second 90 of the UNAIDS 90-90-90 targets, is less than $90 \%$ for those diagnosed with HIV in the province $[4-5,9,19-22]$. Optimal HIV testing and treatment, will only be achieved when the operational and implementation challenges limiting access to care and treatment, often context-specific, are properly addressed ${ }^{[16]}$

There is a dearth of evidence related to the knowledge and perceptions of the HIV services that are offered by the CHWs to PLWH in KZN, therefore this study seeks to find this evidence. Understanding the knowledge and perceptions of PLWH regarding the HIV services offered by CHWs, may provide guidance to both the CHWs and the HIV programme on how to best utilize CHWs in providing effective HIV services to PLWH.

\section{Methods}

\section{Study setting and design}

The study was conducted in three of the seven sub-districts of the uMgungundlovu district in KwaZuluNatal (KZN), the second populous district with a population of $1095865^{\text {[23] }}$. The KZN district has a wellestablished $\mathrm{CHW}$ programme and has a HIV prevalence of $27.9 \%{ }^{[1]}$. To find out the knowledge and perceptions of PLWH regarding the HIV services provided by the $\mathrm{CHWs}$, a descriptive, quantitative survey was conducted amongst PLWH. Purposive sampling was used to select three uMgungundlovu subdistricts and one clinic with the highest HIV prevalence in each sub-district. To calculate the required sample size of 303 , the Cochran formula, $n_{0}=\frac{Z^{2} p q}{e^{2}}$, which is mostly used for large target populations as in this study ${ }^{[24]}$, was used, where $n=$ the sample size that was required in order to generalize the results to the total population; $Z=$ statistic corresponding to $95 \%$ confidence interval (1.96); $p=$ proportion of the phenomenon in the population (HIV prevalence was $27.9 \%$ at KZN $\left.{ }^{[1]}=0.27\right) ; q=1-p(1-0.27)$ and $\mathrm{e}=$ margin of error at $5 \%(0.05)$. Probability proportional to size was used to determine the sample size per clinic.

\section{Data Collection}

People living with HIV that were $\geq 18$ years and routinely come to the clinic to collect their ART treatment were interviewed using an administrator-administered questionnaire. 
Data was analyzed using Social Package of Social Sciences (SPSS), version 27.0. Univariate analysis included frequencies, percentages and means to summarize data. To determine factors associated with knowledge and perceptions of PLWH regarding the HIV services that are offered by the CHWs, bivariate analysis was conducted at $95 \%$ confidence interval $(\mathrm{Cl})$ where a $p$ value of $\leq 0.05$ was considered as statistically significant. The relationship between dichotomous variables and variables with multicategories was tested using odds ratio (OR) and chi-square $\left(\mathbb{\nabla}^{2}\right)$ tests respectively. Pearson's correlation coefficient ( $r$ ) test was used to test the relationship between continuous variables.

\section{Results}

Characteristics of participants

\section{Sociodemographic}

As seen in Table 1., among the 303 participants, 76 (25.1\%) were males and 227 (74.9\%) were females. The median age (inter-quartile range) of participants was 38 years (18-90 years). The mean age for males and females was 42 years (yrs.) and 38 yrs. respectively. Almost a third (32\%) of the study population were between $31-40 \mathrm{yrs}$. of age.

Two thirds (66.6\%) of the study population had between Grade 6-12 level of education [M $=49(24.3 \%)$; $F=153(75.7 \%)]$. There was a statistically significant relationship between gender and the main source of income $(p=0.00 ; 95 \% \mathrm{Cl}: 0.00-0.00)$. Almost half of the men, $(47.3 \%, 36 / 76)$ were employed while most women $[45.8 \%, 104 / 227]$, received government social grant. 
Table 1

Demographics of the study population

\begin{tabular}{|c|c|c|c|c|c|c|}
\hline Variables & Categories & $\begin{array}{l}\text { Male } \\
\mathrm{n}=76 \\
(\%)\end{array}$ & $\begin{array}{l}\text { Female } \\
n=227 \\
(\%)\end{array}$ & $\begin{array}{l}\text { Total } \\
\mathrm{N}=303 \\
(\%)\end{array}$ & $p$-Value & $95 \% \mathrm{Cl}$ \\
\hline \multirow[t]{9}{*}{ Age (yrs.) } & $18-25$ & $7(9.2)$ & $27(11.9)$ & $34(11.2)$ & 0.10 & $\begin{array}{l}0.65- \\
0.13\end{array}$ \\
\hline & $26-30$ & $3(3.9)$ & $31(13.7)$ & $34(11.2)$ & & \\
\hline & $31-40$ & $\begin{array}{l}27 \\
(35.5)\end{array}$ & $69(30.4)$ & $96(31.6)$ & & \\
\hline & $41-50$ & $\begin{array}{l}23 \\
(30.3)\end{array}$ & $44(19.4)$ & $67(22.1)$ & & \\
\hline & $51-60$ & $\begin{array}{l}11 \\
(14.5)\end{array}$ & $31(13.7)$ & $42(13.8)$ & & \\
\hline & $61-70$ & $2(2.6)$ & $18(7.9)$ & $20(6.6)$ & & \\
\hline & $71-80$ & $3(3.9)$ & $6(2.6)$ & $9(2.9)$ & & \\
\hline & $>80$ & $0(0)$ & $1(0.4)$ & $1(0.3)$ & & \\
\hline & Mean Age & 42 yrs. & 38 yrs. & & & \\
\hline \multirow[t]{4}{*}{ Education } & No Education & $9(11.8)$ & $20(8.8)$ & $29(9.6)$ & 0.49 & $\begin{array}{l}0.43- \\
0.53\end{array}$ \\
\hline & Grade $1-5$ & $\begin{array}{l}18 \\
(23.7)\end{array}$ & $49(21.6)$ & $67(22.1)$ & & \\
\hline & Grade $6-12$ & $\begin{array}{l}49 \\
(64.5)\end{array}$ & $\begin{array}{l}153 \\
67.4()\end{array}$ & $\begin{array}{l}202 \\
(66.7)\end{array}$ & & \\
\hline & Post Matric & $0(0.0)$ & $5(2.2)$ & $5(1.7)$ & & \\
\hline \multirow[t]{6}{*}{$\begin{array}{l}\text { Source of } \\
\text { income }\end{array}$} & Employed & $\begin{array}{l}36 \\
(47.4)\end{array}$ & $69(30.4)$ & $\begin{array}{l}105 \\
(34.7)\end{array}$ & $0.00 *$ & $\begin{array}{l}0.00- \\
0.00\end{array}$ \\
\hline & Self-employed & $5(6.6)$ & $6(2.6)$ & $11(3.6)$ & & \\
\hline & $\begin{array}{l}\text { Unemployed (no } \\
\text { source) }\end{array}$ & $\begin{array}{l}10 \\
(13.2)\end{array}$ & $6(2.6)$ & $16(5.2)$ & & \\
\hline & Government grant & $\begin{array}{l}13 \\
(17.1)\end{array}$ & $\begin{array}{l}104 \\
(45.8)\end{array}$ & $\begin{array}{l}117 \\
(38.6)\end{array}$ & & \\
\hline & Sexual partner & $1(1.3)$ & $9(4.0)$ & $10(3.3)$ & & \\
\hline & Parents & $\begin{array}{l}10 \\
(13.2)\end{array}$ & $27(11.9)$ & $37(12.2)$ & & \\
\hline
\end{tabular}




\begin{tabular}{|lllllll|}
\hline Variables & Categories & Male & Female & Total & $p$-Value & $95 \% \mathrm{Cl}$ \\
& $\begin{array}{c}\mathbf{n = 7 6} \\
(\%)\end{array}$ & $\begin{array}{l}\mathbf{n = 2 2 7} \\
(\%)\end{array}$ & $\begin{array}{l}\mathbf{N}=303 \\
(\%)\end{array}$ & \\
\hline & $1(1.3)$ & $6(2.6)$ & $7(2.3)$ & \\
\hline
\end{tabular}

As seen in Table 2., there was a moderate positive linear correlation between age and the duration of visiting the clinic $(r=0.31, p=0.05)$. Older people were more likely to have been visiting the clinic for longer compared to younger people.

Table 2

Pearson Correlation between age and duration of visiting the same clinic

\begin{tabular}{|llll|}
\hline Variables & $\begin{array}{l}\text { Total Sample } \\
(\mathbf{N})\end{array}$ & $\begin{array}{l}\text { Pearson Correlation Coefficient } \\
(\mathbf{r})\end{array}$ & $\boldsymbol{p}$ Value \\
\hline $\begin{array}{l}\text { Age: duration of visiting the same } \\
\text { clinic }\end{array}$ & 303 & 0.31 & 0.05 \\
\hline
\end{tabular}

\section{Community health workers' home visits}

Of the 303 participants, 89 (29.4\%, SD: 0.23) were visited by a CHW [M: 20/76 (26.3\%); F: 69/227 (30.4\%)] in their homes at any time of their lives. Males were less likely to be visited by a CHW compared to females (OR $=0.81, \mathrm{Cl}: 0.46-1.5)$. Furthermore, of the 89,84 (94\%, SD: 0.23) were offered HIV related services by a CHW during a home visit. (Fig. 1.).

The 89 participants visited by the CHWs described the visits as either overall very good $(76.4 \%, 68 / 89)$; good $(20.2 \%, 18 / 89)$ or bad $(3.4 \%, 3 / 89)$. The majority $(93.3 \%, 83 / 89)$ mostly appreciated the HIV related information offered by the $\mathrm{CHW}$, while others liked that the $\mathrm{CHW}$ gave them other health information $(3.4 \%, 3 / 89)$ and helped them with other health issues $(1.1 \%, 1 / 89)$ as seen in Fig. 2.

\section{Knowledge of HIV services offered by CHWs}

There were ten questions with a score of one point for each correct response making the total knowledge score out of ten. Incorrect responses were scored a zero (0).

Participants with a score of nine to ten were categorized as having knowledge of HIV services offered by CHWs.

Participants who scored from zero (0) to eight (8) were categorized as having no knowledge of the HIV services offered by the CHWs.

The mean score was 7.13 (95\% Cl: 6.78-7.48). As seen in Table 3., of the 303 participants, 117 (38.6\%) reported that they knew the HIV services provided by the CHWs at households (Table 2.) However, only 24 $(20.5 \%)$ of them had a knowledge score of nine or ten which indicated that they knew the HIV services that are offered bv the CHWs. 
Overall, eight percent (24/303) of the study population knew about the HIV services provided by the CHWs.

The knowledge of HIV services provided the CHWs was not significantly associated with age $(p=0.63$, 95\% Cl: 0.63-0.7).

However, the majority (37.5\%) of the 24 participants who had knowledge of the HIV services, were from the $31-40$ yrs.

age group who also had higher level of education compared to other age groups. There was a statistically significant relationship between age and education ( $p=0.00,95 \% \mathrm{Cl}: 0.00-0.01)$. Participants who were between 31-60 yrs. had better education than the other age groups. However, education was not associated with the knowledge of HIV services ( $p=0.66,95 \% \mathrm{Cl}: 0.66-0.77)$. 
Table 3

Knowledge of HIV services offered by CHWs

\begin{tabular}{|c|c|c|c|c|c|c|c|}
\hline \multirow[t]{2}{*}{ Variables } & \multirow[t]{2}{*}{ Categories } & \multirow{2}{*}{$\begin{array}{l}\text { KNOW } \\
\text { N=24 } \\
\text { n (\%) }\end{array}$} & \multirow{2}{*}{$\begin{array}{l}\text { DON'T } \\
\text { KNOW } \\
\mathrm{N}=93 \\
\mathrm{n}(\%)\end{array}$} & \multirow{2}{*}{$\begin{array}{l}\begin{array}{l}\text { Total } \\
(\%)\end{array} \\
\mathrm{N}= \\
117\end{array}$} & \multirow[t]{2}{*}{ Value } & \multirow[t]{2}{*}{$\begin{array}{l}\text { Odds } \\
\text { Ratio (OR) }\end{array}$} & \multirow[t]{2}{*}{$\begin{array}{l}95 \% \\
\mathrm{Cl}\end{array}$} \\
\hline & & & & & & & \\
\hline \multirow[t]{8}{*}{ Age (yrs.) } & $18-25$ & $2(8,3)$ & $13(14.0)$ & $\begin{array}{l}15 \\
(12.8)\end{array}$ & 0.63 & - & $\begin{array}{l}0.63- \\
0.74\end{array}$ \\
\hline & $26-30$ & $1(4.2)$ & $9(9.7)$ & $\begin{array}{l}10 \\
(8.5)\end{array}$ & & & \\
\hline & $31-40$ & $\begin{array}{l}9 \\
(37.5)\end{array}$ & $20(21.5)$ & $\begin{array}{l}29 \\
(24.8)\end{array}$ & & & \\
\hline & $41-50$ & $\begin{array}{l}5 \\
(20.8)\end{array}$ & $21(22.6)$ & $\begin{array}{l}26 \\
(22.2)\end{array}$ & & & \\
\hline & $51-60$ & $\begin{array}{l}4 \\
(16.7)\end{array}$ & 17 (18.3) & $\begin{array}{l}21 \\
(17.9)\end{array}$ & & & \\
\hline & $61-70$ & $\begin{array}{l}3 \\
(12.5)\end{array}$ & $9(9.7)$ & $\begin{array}{l}12 \\
(10.3)\end{array}$ & & & \\
\hline & $71-80$ & $0(0.0)$ & $4(4.3)$ & $4(3.4)$ & & & \\
\hline & $>80$ & $0(0.0)$ & $(0.00)$ & $0(0.0)$ & & & \\
\hline \multirow[t]{2}{*}{ Gender } & Male & $\begin{array}{l}6 \\
(25.0)\end{array}$ & $20(21.5)$ & $\begin{array}{l}26 \\
(22.2)\end{array}$ & 0.71 & 1.21 & $\begin{array}{l}0.42- \\
3.47-\end{array}$ \\
\hline & Female & $\begin{array}{l}18 \\
(75.0)\end{array}$ & 73 (78.5) & $\begin{array}{l}91 \\
(77.8)\end{array}$ & & & \\
\hline \multirow[t]{4}{*}{ Education } & No education & $\begin{array}{l}4 \\
(16.7)\end{array}$ & $8(8.6)$ & $\begin{array}{l}12 \\
10.3)\end{array}$ & 0.66 & - & $\begin{array}{l}0.66- \\
0.77\end{array}$ \\
\hline & Grade $1-5$ & $\begin{array}{l}6 \\
(25.0)\end{array}$ & $24(25.8)$ & $\begin{array}{l}30 \\
(25.6)\end{array}$ & & & \\
\hline & Grade 6-12 & $\begin{array}{l}14 \\
(58.3)\end{array}$ & $60(64.5)$ & $\begin{array}{l}74 \\
(63.2)\end{array}$ & & & \\
\hline & Post Matric & $0(0.0)$ & $1(1.1)$ & $1(0.9)$ & & & \\
\hline \multirow[t]{3}{*}{$\begin{array}{l}\text { Source of } \\
\text { income }\end{array}$} & Employed & $\begin{array}{l}4 \\
(16.7)\end{array}$ & $27(29.0)$ & $\begin{array}{l}31 \\
(26.5)\end{array}$ & 0.73 & - & $\begin{array}{l}0.69- \\
0.79\end{array}$ \\
\hline & Self-employed & $0(0.0)$ & $1(1.1)$ & $1(0.9)$ & & & \\
\hline & $\begin{array}{l}\text { Unemployed (no } \\
\text { source) }\end{array}$ & $1(4.2)$ & $7(7.5)$ & $8(6.8)$ & & & \\
\hline
\end{tabular}




\begin{tabular}{|c|c|c|c|c|c|c|c|}
\hline \multirow[t]{5}{*}{ Variables } & Categories & $\begin{array}{l}\text { KNOW } \\
\mathrm{N}=24 \\
\mathrm{n}(\%)\end{array}$ & $\begin{array}{l}\text { DON'T } \\
\text { KNOW } \\
\mathrm{N}=93 \\
\mathrm{n}(\%)\end{array}$ & $\begin{array}{l}\text { Total } \\
(\%) \\
\mathrm{N}= \\
117\end{array}$ & p- & $\begin{array}{l}\text { Odds } \\
\text { Ratio (OR) }\end{array}$ & $\begin{array}{l}95 \% \\
\mathrm{Cl}\end{array}$ \\
\hline & $\begin{array}{l}\text { Government } \\
\text { grant }\end{array}$ & $\begin{array}{l}13 \\
(54.2)\end{array}$ & $40(43.0)$ & $\begin{array}{l}53 \\
(45.3)\end{array}$ & & & \\
\hline & Sexual partner & $2(8.3)$ & $7(7.5)$ & $9(7.7)$ & & & \\
\hline & Parents & $\begin{array}{l}4 \\
(16.7)\end{array}$ & $9(9.7)$ & $\begin{array}{l}13 \\
(11.1)\end{array}$ & & & \\
\hline & Relatives & $0(0.0)$ & $2(2.2)$ & $2(1.7)$ & & & \\
\hline \multirow[t]{5}{*}{$\begin{array}{l}\text { Period visiting } \\
\text { clinic }\end{array}$} & Less than $1 \mathrm{yr}$ & $0(0.0)$ & $2(2.2)$ & $2(1.7)$ & 0.33 & - & $\begin{array}{l}0.21- \\
0.31\end{array}$ \\
\hline & $1 \mathrm{yr}$ & $\begin{array}{l}5 \\
(20.8)\end{array}$ & $7(7.5)$ & $\begin{array}{l}12 \\
(10.3)\end{array}$ & & & \\
\hline & $2-5$ yrs. & $\begin{array}{l}8 \\
(33.3)\end{array}$ & $32(34.4)$ & $\begin{array}{l}40 \\
(34.2)\end{array}$ & & & \\
\hline & $>5 \mathrm{yrs}$ & $\begin{array}{l}11 \\
(45.8)\end{array}$ & $50(53.8)$ & $\begin{array}{l}61 \\
(52.1)\end{array}$ & & & \\
\hline & Since birth & $0(0.0)$ & $2(2.2)$ & $2(1.7)$ & & & \\
\hline \multirow[t]{2}{*}{ Referred } & Yes & $\begin{array}{l}3 \\
(12.5)\end{array}$ & $14(15.1)$ & $\begin{array}{l}17 \\
(14.5)\end{array}$ & 0.75 & 0.80 & $\begin{array}{l}0.21- \\
3.06\end{array}$ \\
\hline & No & $\begin{array}{l}21 \\
(87.5)\end{array}$ & 79 (84.9) & $\begin{array}{l}100 \\
(85.5)\end{array}$ & & & \\
\hline \multirow[t]{2}{*}{$\begin{array}{l}\text { Visited by } \\
\text { CHW }\end{array}$} & Yes & $\begin{array}{l}18 \\
(75.0)\end{array}$ & $61(65.6)$ & $\begin{array}{l}79 \\
(67.5)\end{array}$ & 0.38 & 1.57 & $\begin{array}{l}0.57- \\
4.35\end{array}$ \\
\hline & No & $\begin{array}{l}6 \\
(25.0)\end{array}$ & $32(34.4)$ & $\begin{array}{l}38 \\
(32.5)\end{array}$ & & & \\
\hline
\end{tabular}

Gender was found not associated with knowledge of HIV services offered by CHWs $(p=0.71)$ although males were more likely to know about HIV services offered by CHWs compared to females $(\mathrm{OR}=1.21$, $95 \% \mathrm{Cl}: 0.42-3.47)$. Knowledge of HIV services was not associated with the source of income $(p=0.73$, $95 \% \mathrm{Cl}: 0.69)$ or the duration of visiting the clinic $((p=0.33,95 \% \mathrm{Cl}: 0.21-0.31)$. Referred participants were less likely to know about the HIV services than self-referred participants (OR $=0.80,95 \%$ Cl: $0.21-$ 3.06) although there was no statistically significant relationship between referral and knowledge of the HIV services $(p=0.75)$. There was no significant relationship between being visited by a CHW and knowing the HIV services $(p=0.38)$. 
However, participants that had been visited by a CHW were more likely $(\mathrm{OR}=1.57,95 \% \mathrm{Cl}: 0.57-4.35)$ to know about the HIV services compared to participants who had never been visited by a CHW.

\section{Perceptions about HIV services offered by the community health workers}

Only the participants that were visited by $\mathrm{CHWs}(\mathrm{N}=89)$ were asked about their perceptions of HIV services that they received from a CHW (Table 4). There were five questions that scored one point for each correct response and zero for every incorrect response. The total score was then five (5).

Participants who scored a total of 5 and less than 5 were categorized as having a positive perception and a negative perception respectively. The mean perception score was 4.76 (95\% Cl: 4.62-4.90) 
Table 4

Factors associated with the perception about CHW

\begin{tabular}{|c|c|c|c|c|c|c|c|}
\hline Variables & Categories & $\begin{array}{l}\text { Positive } \\
\text { perception } \\
\mathrm{N}=73 \\
\mathrm{n}(\%)\end{array}$ & $\begin{array}{l}\text { Negative } \\
\text { perception } \mathrm{N} \\
=16 \\
\mathrm{n}(\%)\end{array}$ & $\begin{array}{l}\text { Total } \\
N= \\
89\end{array}$ & $\begin{array}{l}\text { p- } \\
\text { value }\end{array}$ & $\begin{array}{l}\text { Odds } \\
\text { Ratio } \\
\text { (OR) }\end{array}$ & $95 \% \mathrm{Cl}$ \\
\hline \multirow[t]{8}{*}{ Age (yrs.) } & $18-25$ & $10(13.7)$ & $2(12.5)$ & $\begin{array}{l}12 \\
(13.5)\end{array}$ & 0.42 & - & $\begin{array}{l}0.44- \\
0.56\end{array}$ \\
\hline & $26-30$ & $4(5.5)$ & $3(18.8)$ & $\begin{array}{l}7 \\
(7.9)\end{array}$ & & & \\
\hline & $31-40$ & $15(20.5)$ & $1(6.3)$ & $\begin{array}{l}16 \\
(18.0)\end{array}$ & & & \\
\hline & $41-50$ & $14(19.2)$ & 5 (31.0) & $\begin{array}{l}19 \\
(21.3)\end{array}$ & & & \\
\hline & $51-60$ & $16(21.9)$ & $3(18.8)$ & $\begin{array}{l}19 \\
(21.3)\end{array}$ & & & \\
\hline & $61-70$ & $10(13.7)$ & $1(6.3)$ & $\begin{array}{l}11 \\
(12.4)\end{array}$ & & & \\
\hline & $71-80$ & $4(5.5)$ & $1(6.3)$ & $\begin{array}{l}5 \\
(5.6)\end{array}$ & & & \\
\hline & $>80$ & $0(0.0)$ & $0(0.0)$ & $\begin{array}{l}0 \\
(0.0)\end{array}$ & & & \\
\hline \multirow[t]{2}{*}{ Gender } & Male & $18(24.7)$ & $2(12.5)$ & $\begin{array}{l}20 \\
(22.5)\end{array}$ & 0.29 & 2.29 & $\begin{array}{l}0.47- \\
11.05\end{array}$ \\
\hline & Female & $55(75.3)$ & $14(87.5)$ & $\begin{array}{l}69 \\
(77.5)\end{array}$ & & & \\
\hline \multirow[t]{4}{*}{ Education } & $\begin{array}{l}\text { No } \\
\text { education }\end{array}$ & $11(15.1)$ & $0(0.0)$ & $\begin{array}{l}11 \\
(12.4)\end{array}$ & 0.25 & - & $\begin{array}{l}0.29- \\
0.40\end{array}$ \\
\hline & Grade $1-5$ & $23(31.5)$ & $6(37.5)$ & $\begin{array}{l}29 \\
(32.6)\end{array}$ & & & \\
\hline & Grade 6-12 & $39(53.4)$ & $10(62.5)$ & $\begin{array}{l}49 \\
(55.1)\end{array}$ & & & \\
\hline & Post Matric & $0(0.0)$ & $0(0.0)$ & & & & \\
\hline \multirow[t]{2}{*}{$\begin{array}{l}\text { Source of } \\
\text { income }\end{array}$} & Employed & $18(24.7)$ & $2(12.5)$ & $\begin{array}{l}20 \\
(22.5)\end{array}$ & 0.73 & - & $\begin{array}{l}0.74- \\
0.83\end{array}$ \\
\hline & $\begin{array}{l}\text { Self- } \\
\text { employed }\end{array}$ & $1(1.4)$ & $1(6.3)$ & $\begin{array}{l}2 \\
(2.2)\end{array}$ & & & \\
\hline
\end{tabular}




\begin{tabular}{|c|c|c|c|c|c|c|c|}
\hline Variables & Categories & $\begin{array}{l}\text { Positive } \\
\text { perception } \\
\mathrm{N}=73 \\
\mathrm{n}(\%)\end{array}$ & $\begin{array}{l}\begin{array}{l}\text { Negative } \\
\text { perception } \mathrm{N}\end{array} \\
=16 \\
\mathrm{n}(\%)\end{array}$ & $\begin{array}{l}\text { Total } \\
\mathrm{N}= \\
89\end{array}$ & $\begin{array}{l}\mathrm{p}- \\
\text { value }\end{array}$ & $\begin{array}{l}\text { Odds } \\
\text { Ratio } \\
\text { (OR) }\end{array}$ & $95 \% \mathrm{Cl}$ \\
\hline & $\begin{array}{l}\text { Unemployed } \\
\text { (no source) }\end{array}$ & $4(5.5)$ & $0(0.0)$ & $\begin{array}{l}4 \\
(4.5)\end{array}$ & & & \\
\hline & $\begin{array}{l}\text { Government } \\
\text { grant }\end{array}$ & 35 (47.9) & $9(56.3)$ & $\begin{array}{l}44 \\
(49.4)\end{array}$ & & & \\
\hline & $\begin{array}{l}\text { Sexual } \\
\text { partner }\end{array}$ & $5(6.8)$ & $1(6.3)$ & $\begin{array}{l}6 \\
(6.7)\end{array}$ & & & \\
\hline & Parents & $7(9.6)$ & $2(12.5)$ & $\begin{array}{l}9 \\
(10.1)\end{array}$ & & & \\
\hline & Relatives & $3(4.1)$ & $1(6.3)$ & $\begin{array}{l}4 \\
(4.5)\end{array}$ & & & \\
\hline \multirow[t]{5}{*}{$\begin{array}{l}\text { Duration visiting } \\
\text { clinic }\end{array}$} & $\begin{array}{l}\text { Less than } \\
1 \text { year. }\end{array}$ & $1(1.4)$ & $0(0.0)$ & I (1.1) & 0.90 & - & $\begin{array}{l}0.82- \\
0.9\end{array}$ \\
\hline & 1 year. & $6(8.2)$ & $2(1.5)$ & $\begin{array}{l}8 \\
(9.0)\end{array}$ & & & \\
\hline & $2-5 \mathrm{yrs}$ & $27(37.0)$ & $7(43.8)$ & $\begin{array}{l}34 \\
(38.2)\end{array}$ & & & \\
\hline & $>5 \mathrm{yrs}$. & 38 (52.1) & $7(43.8)$ & $\begin{array}{l}45 \\
(50.6)\end{array}$ & & & \\
\hline & Since birth & $1(1.4)$ & $0(0.0)$ & $\begin{array}{l}1 \\
(1.1)\end{array}$ & & & \\
\hline \multirow[t]{2}{*}{ Referred } & Yes & $13(17.8)$ & $4(25.0)$ & $\begin{array}{l}17 \\
(19.1)\end{array}$ & 0.50 & 0.65 & $\begin{array}{l}0.18- \\
2.33\end{array}$ \\
\hline & No & 60 (82.2) & $12(75.0)$ & $\begin{array}{l}72 \\
(80.9)\end{array}$ & & & \\
\hline \multirow[t]{2}{*}{$\begin{array}{l}\text { Know about HIV } \\
\text { services }\end{array}$} & Yes & $18(27.7)$ & $0(0 / 0)$ & $\begin{array}{l}18 \\
(22.8)\end{array}$ & $0.03^{*}$ & 1.3 & $\begin{array}{l}1.13- \\
1.48\end{array}$ \\
\hline & No & 47 (72.3) & $14(100.0)$ & $\begin{array}{l}61 \\
(77.2)\end{array}$ & & & \\
\hline \multirow[t]{2}{*}{$\begin{array}{l}\text { CHWs are not } \\
\text { educated }\end{array}$} & Yes & $2(2.7)$ & $4(25.0)$ & $\begin{array}{l}6 \\
(6.7)\end{array}$ & $0.01 *$ & 0.85 & $\begin{array}{l}0.14- \\
0.51\end{array}$ \\
\hline & No & 71 (97.3) & $12(75.0)$ & $\begin{array}{l}83 \\
(93.3)\end{array}$ & & & \\
\hline $\begin{array}{l}\text { CHWs do not } \\
\text { know about HIV }\end{array}$ & Yes & $0(0.0)$ & $1(6.3)$ & $\begin{array}{l}1 \\
(1.1)\end{array}$ & $0.03^{*}$ & 5.87 & $\begin{array}{l}3.7- \\
9.3\end{array}$ \\
\hline
\end{tabular}

Loading [MathJax]/jax/output/CommonHTML/fonts/TeX/fontdata.js 


\begin{tabular}{|c|c|c|c|c|c|c|c|}
\hline Variables & Categories & $\begin{array}{l}\text { Positive } \\
\text { perception } \\
\mathrm{N}=73 \\
\mathrm{n}(\%)\end{array}$ & $\begin{array}{l}\text { Negative } \\
\text { perception } \mathrm{N} \\
=16 \\
\mathrm{n}(\%)\end{array}$ & $\begin{array}{l}\text { Total } \\
N= \\
89\end{array}$ & $\begin{array}{l}\mathrm{p}- \\
\text { value }\end{array}$ & $\begin{array}{l}\text { Odds } \\
\text { Ratio } \\
\text { (OR) }\end{array}$ & $95 \% \mathrm{Cl}$ \\
\hline & No & $73(100.0)$ & $1(6.3)$ & $\begin{array}{l}88 \\
(98.9)\end{array}$ & & & \\
\hline \multirow[t]{2}{*}{$\begin{array}{l}\text { CHWs lack } \\
\text { confidentiality }\end{array}$} & Yes & $0(0.0)$ & $14(87.5)$ & $\begin{array}{l}14 \\
(15.7)\end{array}$ & $0.00 *$ & 37.0 & $\begin{array}{l}9.5- \\
147.17\end{array}$ \\
\hline & No & $73(100.0)$ & $2(12.5)$ & $\begin{array}{l}75 \\
(84.3)\end{array}$ & & & \\
\hline \multirow[t]{2}{*}{$\begin{array}{l}\text { CHW answered } \\
\text { all my questions }\end{array}$} & Yes & $72(98.6)$ & 13 (81.3) & $\begin{array}{l}85 \\
(95.5)\end{array}$ & $0.02^{*}$ & 16.15 & $\begin{array}{l}(1.60- \\
172.0)\end{array}$ \\
\hline & No & $1(1.4)$ & $3(18.8)$ & $\begin{array}{l}4 \\
(4.5)\end{array}$ & & & \\
\hline \multirow[t]{2}{*}{$\begin{array}{l}\text { CHWs services } \\
\text { helpful }\end{array}$} & Yes & $73(100.0$ & 14 (87.5) & $\begin{array}{l}87 \\
(97.8)\end{array}$ & $0.02 *$ & 0.16 & $\begin{array}{l}0.1- \\
0.26\end{array}$ \\
\hline & No & $0(0.0)$ & $2(12.5)$ & $\begin{array}{l}2 \\
(2.2)\end{array}$ & & & \\
\hline
\end{tabular}

\section{*significant results}

Of the 89 participants visited by a CHW, 73 (82\%) and $16(18 \%)$ had positive and negative perceptions about HIV services offered by the $\mathrm{CHWs}$ respectively.

Age $(p=0.42,95 \% \mathrm{Cl}: 0.44-0.56)$, gender $(p=0.29, \mathrm{OR}=2.29,95 \% \mathrm{Cl}: 0.47-11.05)$, education $((p=0.25$, $95 \% \mathrm{Cl}: 0.29-0.40)$, source of income $(p=0.73,95 \% \mathrm{Cl}: 0.74-0.83)$ and period visiting the clinic $(p=0.90$, 95\% Cl: 0.82-0.9) were not associated with the way PLWH perceived the HIV services offered by the $\mathrm{CHWs}$. Those who were referred by a doctor, nurse or $\mathrm{CHW}$ were less likely to have positive perception of CHWs (OR $=0.65,95 \% \mathrm{Cl}: 0.18-2.33)$ compared to those who self-referred. Knowledge of HIV services was significantly associated with with the perception of HIV services offered by CHWs $(p=0.03,95 \% \mathrm{Cl}$ : 1.13-1.48), whereby those who knew about the HIV services offered by the CHWs were most likely to have a positive perception about these services $(\mathrm{OR}=1.3 .95 \% \mathrm{Cl}: 1.13-1.48)$. Participants who perceived that the $\mathrm{CHWs}$ lacked confidentiality, were most likely to have a negative perception towards the HIV services that were rendered by $\mathrm{CHWs}(p=0.00 ; \mathrm{OR}=37.0, \mathrm{Cl}: 9.5-172)$.

\section{Discussion}

This study sought to determine the knowledge and perceptions of PLWH regarding the HIV services that are offered by the CHWs in the KZN province. 
The study found that the majority of PLWH do not know about the HIV services that are provided the $\mathrm{CHWs}$ and less than a third of them have received HIV services offered by CHWs in their homes. In addition, the study found that PLWH are more likely to know and subsequently have a positive perception towards the HIV services that are offered by CHWs if they were visited by $\mathrm{CHWs}$. These findings are similar to other studies conducted in KZN and in other similar settings, where CHW visits were associated with good HIV knowledge ${ }^{[25-26]}$. Regarding our finding that some of the PLWH have negative perceptions towards the HIV services offered by the $\mathrm{CHW}$, a systematic review conducted in Middle East and North African countries, showed that in general, PLWH have low knowledge and have a negative attitude towards HIV prevention and and treatment interventions [27].

The odds of knowing about the HIV services offered by the CHWs, were higher among those who received $\mathrm{CHW}$ visits than those who were never visited by a CHW. However, the majority of PLWH in KZN have never been visited by a CHW and therefore are less likely to know about the HIV services that CHWs provide and from which they can benefit. Similar findings were found in KZN and other similar settings, where the uptake of CHW- led HIV services was low due to a variety of reasons including the health system related barriers that CHW were faced with, HIV stigma, community and political contexts [26, 28-31]. In addition, the study found that the minority that ever received a CHW visit, were not all offered HIV related services by the CHW. This indicates missed opportunities of HIV education and treatment for HIV in the province. This is in spite of the evidence that the uptake of HIV treatment reduces HIV transmission $[4,16,18,32]$. The low numbers of CHW-referrals may also be linked to the province's poor and delayed linkage to care for people that have tested positive and referred for HIV testing and treatment by the home based care programmes ${ }^{[33-36]}$.

The majority of PLWH who were visited by CHWs, mostly had positive perceptions towards the HIV services offered by $\mathrm{CHWs}$ while the minority had negative perceptions. Negative perceptions towards HIV services offered by $\mathrm{CHWs}$ were due to the idea that $\mathrm{CHWs}$ are not well educated, are not well informed about HIV but mostly that they lacked confidentiality. This finding is consistent with other studies that were conducted in KZN and South Africa [11,37-39]]. Perceptions of poor confidentiality has diminished trust towards $\mathrm{CHWs}$ and have been associated with negative attitudes and low acceptance of this cadre [11,37-39]. Furthermore, the communities in KZN have been reported to disrespect $\mathrm{CHWs}$ and have a perception that they lack professionalism compared to professional nurses ${ }^{[40]}$. Although education and source of income have been found to be significantly associated with a positive response towards HIV services offered by the $\mathrm{CHWs}{ }^{[2,9]}$, in this study education and employment were not associated with any perception of the same services.

The sampling method, using a statistical formula contributed to the strength of the study and therefore the study findings can be generalized to the entire population of KZN. However, sampling was from only one of the eleven KZN districts which may pose bias to the study. In future, sampling for a similar study may need to be from other districts in KZN. 
In conclusion, the study results suggest that the majority of PLWH in KZN are not benefiting from the HIV services offered by the CHWs and have a negative attitude towards these services. The study findings should trigger the scaling-up of HIV community targeted initiatives that are delivered by the CHWs in order to curb the HIV epidemic in the province.

\section{Recommendations}

A CHW intervention is most effective when implemented within a carefully defined target population and when this population has been educated about the programme to understand the roles and tasks of $\mathrm{CHWs}^{[41]}$. The provincial $\mathrm{CHW}$ programme planners and policy makers, may need to implement the Intervention model designed by Masquiller et al. of a CHW-led intervention as the way to improve knowledge and acceptance of CHW-HIV services among PLWH ${ }^{[42]}$. In addition, $\mathrm{CHWs}$ may use the existing community health promotion campaigns and home visits as a platform platform to educate and gain trust among communities regarding the HIV services that they provide.

Furthermore, $\mathrm{CHWs}$ should be encouraged to be part of the community peer and youth groups for the formulation of trust and lasting relationships with the communities. When they are trusted by the communities, the perception of the HIV services that they offer can also improve. The perception of HIV services offered by CHWs can also be improved by providing knowledge about these services through pamphlets, community radio stations and routine health promotion campaigns.

\section{Conclusion}

There is increasing reliance on and utilization of $\mathrm{CHWs}$ to provide chronic disease management among PLWH but evidently the majority of PLWH are not benefiting from this service. Moreover, PLWH are ignorant of HIV services offered by $\mathrm{CHWs}$ and some have a lack of trust for $\mathrm{CHWs}$. The study provides a catalyst for investigating barriers to $\mathrm{CHWs}$ visits in future research.

\section{Abbreviations}

ART Antiretroviral treatment

CHW community health worker

CHWs community health workers

HIV Human Immunodeficiency Virus

KZN KwaZulu-Natal

KZN-DoH KwaZulu-Natal-Department of Health 
PLWH People living with HIV

SPSS Statistical Package for Social Sciences

UNAIDS United Nations Programme on HIV/AIDS

UTT Universal test and Treat

WBPHCOT Ward Based Primary Health Care Outreach Team

\section{Declarations}

\section{Ethics approval and consent to participate}

The study was conducted according to the South African Good Clinical Practice Guidelines and the National Health Act (61/2003): Regulations relating to research with human participants (R.719). People living with HIV are considered as a vulnerable group hence participants were assured of anonymity and confidentiality. The study was explained verbally to the study participants and an information sheet given to them for their own perusal. The participants were informed that the study was voluntary and were not coerced to participate in the study. Thereafter, informed consent was obtained from the participant by signing the consent form prior to data collection. The study was approved by the Biomedical Research Ethics Committee of the University of KZN under the reference number BREC 00000765/2019. Clearance for accessing PLWH in the clinics was obtained from the PHC programme manager, the uMgungundlovu District Manager and the KZN-DoH, Health Research and Ethics Committee under reference KZ_202002_008.

\section{Consent for publication}

No person's name were used in this manuscript

\section{Data availability statement}

The datasets used and/or analysed during the current study are available from the corresponding author on reasonable request.

\section{Competing interests}

The authors declare that they have no competing interest.

\section{Funding Details}

The study did not receive any funding.

\section{Author contribution}


GEK wrote the study proposal with the assistance of EL and TPM. BS assisted GEK with data collection data capturing. GEK drafted the manuscript. EL, BS \& TPM substantially provided input towards the draft manuscript until the final draft. All authors read and approved the final manuscript.

\section{Acknowledgements}

We wish to thank the University of KwaZulu-Natal and the KZN Department of Health for supporting the study.

\section{References}

1. Kharsany ABM, Cawood C, Khanyile D, et al. Community-based HIV prevalence in KwaZulu-Natal, South Africa: results of a cross-sectional household survey. Lancet HIV 2018;5(8):e427-e37.

2. Ramjee G, Daniels B. Women and HIV in Sub-Saharan Africa. AIDS Research and Therapy 2013;10(1):30. doi: 10.1186/1742-6405-10-30

3. Shamu S, Chasela C, Slabbert J, et al. Social franchising of community-based HIV counselling and testing services to increase HIV testing and linkage to care in Tshwane, South Africa: study protocol for a non-randomised implementation trial. BMC public health 2020;20(1):118. doi: 10.1186/s12889020-8231-x

4. Johnson LF, Dorrington RE, Moolla H. Progress towards the 2020 targets for HIV diagnosis and antiretroviral treatment in South Africa. 20172017;18(1) doi: 10.4102/sajhivmed.v18i1.694 [published Online First: 2017-01-31]

5. Loeliger KB, Niccolai LM, Mtungwa LN, et al. "I Have to Push Him with a Wheelbarrow to the Clinic": Community Health Workers' Roles, Needs, and Strategies to Improve HIV Care in Rural South Africa. AIDS Patient Care STDS 2016;30(8):385-94. doi: 10.1089/apc.2016.0096 [published Online First: 2016/08/11]

6. Ndaba T, Taylor M, Mabaso M. Training and evaluation of community health workers (CHWs): towards improving maternal and newborn survival in an urban setting in KwaZulu-Natal, South Africa. The Open Public Health Journal 2019;12(1)

7. Naidoo N, Matlakala N, Railton J, et al. Provision of HIV services by community health workers should be strengthened to achieve full programme potential: a cross-sectional analysis in rural South Africa. Tropical Medicine \& International Health: TM \& IH2019;24(4):401-08. doi: 10.1111/tmi.13204

8. Loeliger KB, Niccolai LM, Mtungwa LN, et al. Antiretroviral therapy initiation and adherence in rural South Africa: community health workers' perspectives on barriers and facilitators. AIDS Care 2016;28(8):982-93. doi: 10.1080/09540121.2016.1164292 [published Online First: 2016/04/05]

9. Baisley K, Chimbindi N, Mthiyane N, et al. High HIV incidence and low uptake of HIV prevention services: The context of risk for young male adults prior to DREAMS in rural KwaZulu-Natal, South Africa. PLOS ONE 2018;13(12):e0208689. doi: 10.1371/journal.pone.0208689 
10. Celletti F, Wright A, Palen J, et al. Can the deployment of community health workers for the delivery of HIV services represent an effective and sustainable response to health workforce shortages? Results of a multicountry study. AIDS (London, England) 2010;24 Suppl 1:S45-S57. doi:

10.1097/01.aids.0000366082.68321.d6

11. Grant M, Wilford A, Haskins L, et al. Trust of community health workers influences the acceptance of community-based maternal and child health services. Afr J Prim Health Care Fam Med 2017;9(1):e1e8. doi: 10.4102/phcfm.v9i1.1281 [published Online First: 2017/06/07]

12. Kubheka SE, Archary M, Naidu KK. HIV viral load testing coverage and timeliness after implementation of the wellness anniversary in a paediatric and adolescent HIV clinic in KwaZuluNatal, South Africa. Southern African Journal of HIV Medicine 2020;21:1-5.

13. Daviaud E, Besada D, Budlender D, et al. Saving lives, saving costs: investment case for community health workers in South Africa. Cape Town: South African Medical Research Council 2018

14. Department of Health South Africa. Policy Framework and Strategy for Ward Based Primary Healthcare Outreach Teams (2018/9- 2023/2024). In: Department of Health South Africa, ed. Pretoria, 2018.

15. Department of Heallth KwaZulu-Natal. Universal Test and Treat Pietermaritzburg2016 [Available from: http://www.kznhealth.gov.za/test_treat.htm accessed 01 September 2020.

16. Iwuji CC, Orne-Gliemann J, Larmarange J, et al. Universal test and treat and the HIV epidemic in rural South Africa: a phase 4, open-label, community cluster randomised trial. The lancet HIV 2018;5(3):e116-e25. doi: 10.1016/S2352-3018(17)30205-9

17. Larmarange J. From individual care trajectories to HIV care cascade at population level in rural KwaZulu-Natal (South Africa): the impact of population dynamics-Joseph Larmarange. 2020

18. Larmarange J, Diallo MH, McGrath $\mathrm{N}$, et al. Temporal trends of population viral suppression in the context of Universal Test and Treat: the ANRS 12249 TasP trial in rural South Africa. Journal of the International AIDS Society 2019;22(10):e25402. doi: 10.1002/jia2.25402

19. Vandormael A, Cuadros D, Kim H-Y, et al. The state of the HIV epidemic in rural KwaZulu-Natal, South Africa: a novel application of disease metrics to assess trajectories and highlight areas for intervention. International Journal of Epidemiology 2020;49(2):666-75. doi: 10.1093/ije/dyz269

20. Adeagbo 0 , Herbst $C$, Blandford A, et al. Exploring people's candidacy for mobile health-supported HIV testing and care services in rural KwaZulu-Natal, South Africa: Qualitative study. Journal of medical Internet research 2019;21(11):e15681.

21. Bell J, Sharma S, Malone S, et al. Understanding barriers to HIV testing and treatment: a study of young men and healthcare providers in KwaZulu-Natal and Mpumalanga. South African Health Review 2019;2019(1):125-32.

22. Chimbindi N, Mthiyane N, Birdthistle I, et al. Persistently high incidence of HIV and poor service uptake in adolescent girls and young women in rural KwaZulu-Natal, South Africa prior to DREAMS. PLOS ONE 2018;13(10):e0203193. doi: 10.1371/journal.pone.0203193 
23. Statistics South Africa. Community Survey 2016: provincial Profile, KwaZulu-Natal Pretoria South Africa,2018 [cited 201920 August]. Available from: http://cs2016.statssa.gov.za/wpcontent/uploads/2018/07/KZN.pdf.

24. Cochran WG. Sampling techniques: John Wiley \& Sons 2007.

25. Horwood C, Butler L, Barker P, et al. A continuous quality improvement intervention to improve the effectiveness of community health workers providing care to mothers and children: a cluster randomised controlled trial in South Africa. Hum Resour Health 2017;15(1):39. doi: 10.1186/s12960017-0210-7 [published Online First: 2017/06/15]

26. McCollum R, Otiso L, Mireku M, et al. Exploring perceptions of community health policy in Kenya and identifying implications for policy change. Health Policy Plan 2016;31(1):10-20. doi:

10.1093/heapol/czv007 [published Online First: 2015/03/31]

27. Mumtaz GR, Hilmi N, Majed EZ, et al. Characterising HIV/AIDS knowledge and attitudes in the Middle East and North Africa: Systematic review and data synthesis. Global Public Health 2020;15(2):27598.

28. Kalonji D, Mahomed $\mathrm{OH}$. Health system challenges affecting HIV and tuberculosis integration at primary healthcare clinics in Durban, South Africa. Afr J Prim Health Care Fam Med 2019;11(1):e1e7. doi: 10.4102/phcfm.v11i1.1831 [published Online First: 2019/06/07]

29. Krishnaratne S, Bond V, Stangl A, et al. Stigma and Judgment Toward People Living with HIV and Key Population Groups Among Three Cadres of Health Workers in South Africa and Zambia: Analysis of Data from the HPTN 071 (PopART) Trial. AIDS Patient Care and STDs 2020;34(1):38-50.

30. Mhlongo EM, Lutge E. The roles, responsibilities and perceptions of community health workers and ward-based primary health care outreach teams (WBPHCOTs) in South Africa: a scoping review protocol. Syst Rev 2019;8(1):193. doi: 10.1186/s13643-019-1114-5 [published Online First: 2019/08/07]

31. van Rooyen $\mathrm{H}$, Makusha $\mathrm{T}$, Joseph $\mathrm{P}$, et al. Zwakala Ndoda: a cluster and individually randomized trial aimed at improving testing, linkage, and adherence to treatment for hard-to reach men in KwaZulu-Natal, South Africa. Trials 2019;20(1):1-14.

32. Larmarange J. Entry into care following universal home-based HIV testing in rural KwaZulu-Natal, South Africa: the ANRS TasP 12249 cluster-randomised trial-Joseph Larmarange. 2020

33. Baisley K, Seeley J, Siedner M, et al. Findings from home-based HIV testing and facilitated linkage after scale-up of test and treat in rural South Africa: young people still missing. HIV medicine 2019;20(10):704-08.

34. Iwuji CC, Orne-Gliemann J, Larmarange J, et al. Uptake of home-based HIV testing, linkage to care, and community attitudes about ART in rural KwaZulu-Natal, South Africa: descriptive results from the first phase of the ANRS 12249 TasP cluster-randomised trial. PLoS medicine 2016;13(8):e1002107.

35. Maughan-Brown B, Beckett S, Kharsany AB, et al. Poor rates of linkage to HIV care and uptake of diagnosed 15-to-49 year-old men and women 
in a high HIV prevalence setting in South Africa. AIDS care 2020:1-10.

36. Plazy M, Farouki KE, Iwuji C, et al. Access to HIV care in the context of universal test and treat: challenges within the ANRS 12249 TasP cluster-randomized trial in rural South Africa. Journal of the International AIDS Society 2016;19(1):1-12. doi: 10.7448/IAS.19.1.20913

37. Mlotshwa $L$, Harris $B$, Schneider $\mathrm{H}$, et al. Exploring the perceptions and experiences of community health workers using role identity theory. Glob Health Action 2015;8:28045-45. doi: 10.3402/gha.v8.28045

38. Ngcobo S, Rossouw T. "They come and knock at the gate until the neighbours see". Perceived barriers and benefits of implementing HIV care at the community level in Tshwane district: A qualitative study. PLOS ONE 2020;15(10):e0240740. doi: 10.1371/journal.pone.0240740

39. White MS, Govender P, Lister HE. Community health workers lensed through a South African backdrop of two peri-urban communities in KwaZulu-Natal. 20172017;6 doi: 10.4102/ajod.v6i0.294 [published Online First: 2017-01-31]

40. Mottiar S, Lodge T. The role of community health workers in supporting South Africa's HIV/ AIDS treatment programme. Afr J AIDS Res 2018;17(1):54-61. doi: 10.2989/16085906.2017.1402793 [published Online First: 2018/03/06]

41. World Heath Organization. Essential prevention and care interventions for adults and adolescents living with HIV in resource-limited settings Geneva, Switzerland: WHO, 2008.

42. Masquillier $C$, Wouters $E$, Campbell $L$, et al. Households in HIV care: designing an intervention to stimulate HIV competency in households in South Africa. Frontiers in Public Health 2020;8:246.

\section{Figures}

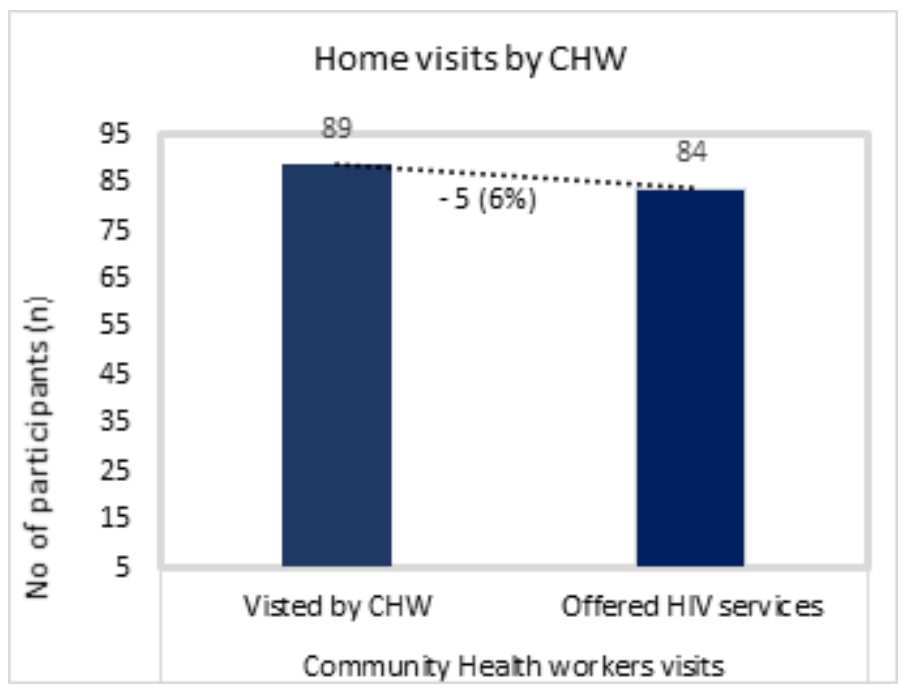

\section{Figure 1}

Participants visited by CHWs and number of participants offered HIV services 


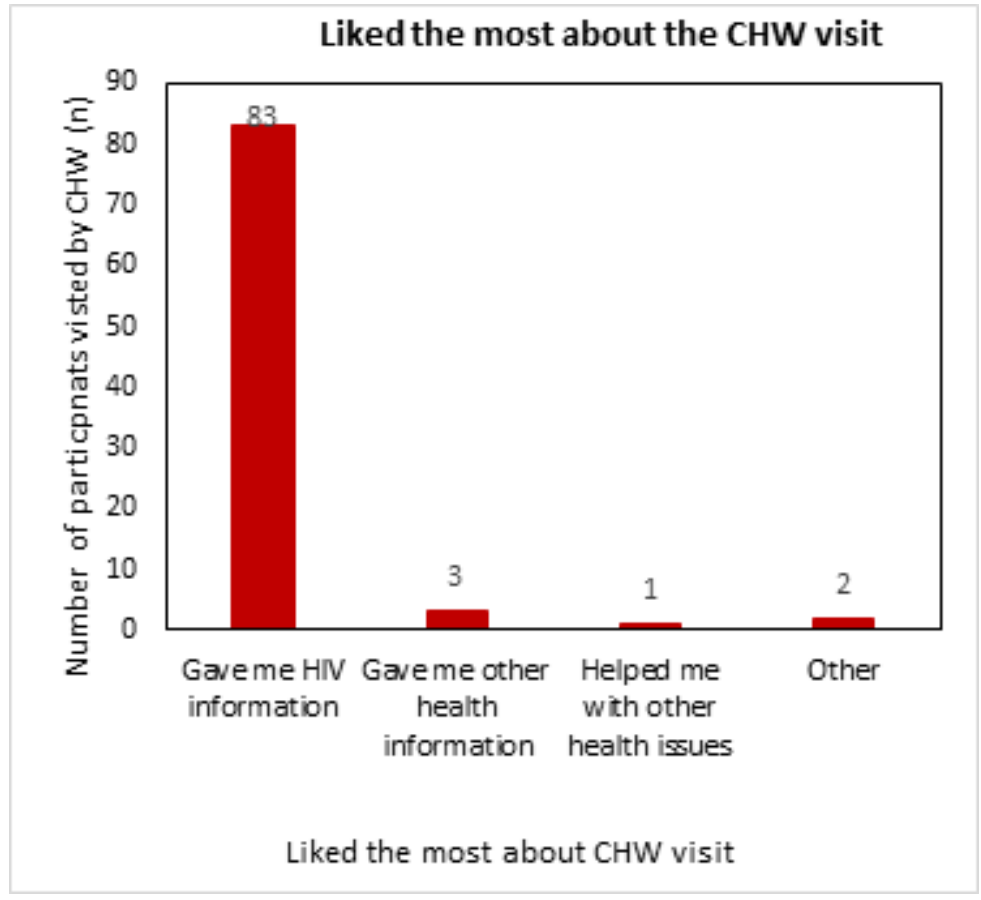

Figure 2

What the communities most liked about $\mathrm{CHW}$ visit 